\title{
«EL CAFÉ TIENE CAFEÍNA Y NOS DESPIERTA, NOS DA ENERGÍA»: CONCEPCIONES SOBRE LA ENERGÍA QUÍMICA, UNA BUENA RAZÓN PARA PONER DE ACUERDO A LOS PROFESORES DE FÍSICA Y QUÍMICA Y CIENCIAS NATURALES
}

\author{
GALLÁSTEGUI OTERO, J.R. ${ }^{1}$ Y LORENZO BARRAL, F.M. ${ }^{2}$ \\ ${ }^{1}$ Instituto de Educación Secundaria e Profesional «Xulián Magariños». Negreira (A Coruña). \\ ${ }^{2}$ Departamento de Didáctica das Ciencias Experimentais. Universidade de Santiago.
}

\section{SUMMARY}

A research was carried on students' conceptions about chemical energy. The experimental sample were students from primary level to university. Word association tasks and a paper and pencil question were used as instruments for collecting data. The test revealed that some students share certain alternative conceptions about chemical energy, and that most of them use ideas about chemical energy in a similar way to what people without formal instruction in this issue would do. Some implications for science teaching-learning are suggested.

\section{INTRODUCCIÓN}

La Energía es uno de los conceptos que mejor podemos calificar como transversal dentro del currículo. En todos los niveles de la enseñanza aparece en diferentes áreas, núcleos, bloques o temas. El hecho de que uno de los bloques del Diseño Curricular Base de Ciencias de secundaria obligatoria sea «La Energía» confirma la importancia curricular de este aspecto. Existen en la bibliografía numerosos trabajos que muestran las dificultades que tienen los alumnos y alumnas que aprenden Ciencias con el concepto de energía y los aspectos relacionados con él, como la conservación de la energía o su degradación (Welch 1986, Brook 1986 y Pintó Casulieras 1991).

Las dificultades de los estudiantes a la hora de reconocer la energía dependen también, en gran medida, de la forma de energía de que se trate. Las energías de tipo mecánico, como la cinética o la potencial gravitatoria, son reconocidas con mayor facilidad: está claro que una piedra que está elevada a una cierta altura sobre el suelo o un tren que va a $120 \mathrm{~km} / \mathrm{h}$ tienen «algo» que es fácil de entender, aunque después pueda haber problemas a la hora de generalizar ese «algo» como la propia energía.

En cambio, la energía que suele llamarse de tipo químico, es decir, aquelia asociada a la estructura química de las sustancias, no es entendida ni intuida con la misma facilidad. Y ello a pesar de la enorme importancia que tiene en la vida diaria y de la frecuencia con que aparece en el currículo: la energía química almacenada en los combustibles (combustión como proceso químico, disponibilidad de recursos, educación para el medio ambiente...) o en los alimentos (alimentación y nutrición: educación para la salud), cuantificada en las etiquetas de los productos alimenticios (educación para el consumo), nos rodea por todas partes, y sin embargo a los alumnos y alumnas les resulta difícil entender cómo algunas sustancias por «ser ellas quienes son» pueden contener energía.

Esta dificultad de los alumnos para reconocer energías de tipo químico aparece implícitamente reconociđa en el siguiente texto, extraído de materiales curriculares sobre la energía para la educación secundaria de reciente publicación (Dapena Márquez et al. 1991):

Una masa de agua a cierta altura posee energía.

Un trozo de carbón también tiene energía porque al quemarlo produce calor.

(EI resaltado es nuestro.)

Los autores sienten la necesidad de "demostrar" la existencia de energía en el carbón, relacionándola con aigo tan evidente como el calor. Sin embargo la energía de la masa de agua a cierta altura no necesita ser demostrada. 
En Biología, una de las mayores dificultades detectadas en el aprendizaje de la fotosíntesis es que muchas veces los estudiantes entienden dicho proceso como un mero intercambio de gases y no reconocen la parte más importante, como es la producción, por parte de las plantas que la realizan, de materia orgánica con alto contenido energético: los glúcidos.

Si la energía de tipo químico aparece en los programas de Física y Química, por un lado, y en los de Biología, porotro, y los alumnos tienen dificultades en la comprensión y utilización de este concepto, ino nos supone un reto a los profesores de Física y Química y a los de Ciencias Naturales?

Por las razones que acabamos de exponer, en este trabajo llevamos a cabo una investigación sobre concepciones de los alumnos acerca de la energía química, partiendo para ello de dos hipótesis:

- Los alumnos tienen dificultades de comprensión de la energía química e ideas alternativas sobre ella.

- Los alumnos, después de recibir instrucción, ponen menos énfasis en la energía de tipo químico que en otros tipos de energía.

\section{MÉTODO}

Para realizar un diagnóstico de la situación de las ideas de los alumnos y alumnas sobre la energía química, se han realizado dos tipos de prueba: un test de asociación libre de palabras utilizando como estímulo la palabra "energía», y un test de lápiz y papel con una pregunta con una parte cerrada y una parte abierta.

En el test de asociación libre de palabras se pidió a los alumnos que escribieran palabras relacionadas con dicho término durante una clase de ciencias (lo que en cierta medida marca el contexto), durante un tiempo de dos minutos (Shavelson 1974).

Como muestra para este test se eligieron dos grupos de Magisterio, que ya habían recibido instrucción en ciencias durante su educación primaria y secundaria. En la tabla I se recogen los datos acerca de los alumnos y alumnas que realizaron la prueba.

Tabla I

Alumnos y alumnas que realizaron la prueba de asociación libre de palabras.

NIVEL CURSO ESPECIALIDAD EDAD N

Universidad

$\begin{array}{lclll}\text { (EU Magisterio) } & 3^{9} & \text { Ciencias } & 20-23 & 40 \\ \begin{array}{l}\text { Universidad } \\ \text { (EU Magisterio) }\end{array} & 3^{9} & \text { Preescolar } & 20-23 & 52\end{array}$

La segunda parte de la investigación, el test de lápiz y papel con una pregunta con una parte cerrada y una parte abierta, se presentó a una muestra de estudiantes de $8^{\circ}$ de EGB, $2^{\circ}$ de BUP, Magisterio y licenciados en Química que estaban cursando el CAP (Tabla II) en una hoja cuyo contenido refleja la figura 1 .

Tabla II

Alumnos y alumnas que contestaron la pregunta con una parte cerrada y una parte abierta del test de lápiz y papel.

\begin{tabular}{llccr}
\hline NIVEL & CURSO & ESPECIALIDAD & EDAD & $\mathrm{N}$ \\
& & & $\vdots$ & \\
EGB & $8^{2}$ & - & $13-14$ & 101 \\
BUP & $2^{\circ}$ & - & $15-16$ & 22 \\
Universidad & & & & \\
(EU Magisterio) & $3^{\circ}$ & Ciencias & $20-23$ & 44 \\
Licenciados & CAP & Química & $>22$ & 16 \\
\hline
\end{tabular}

Figura 1

Test de lápiz y papel.

\section{ALUMNO}

CENTRO

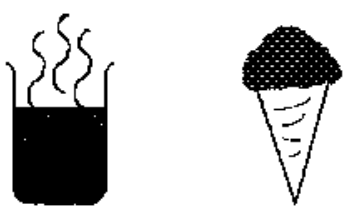

¿Qué crees que aporta más energía a tu organismo: un vaso de café muy caliente o un helado?

Explícalo.

A la hora de valorar el aporte energético a nuestro organismo del café o del helado es necesario especificar una serie de variables que no se dan en el enunciado: cuánta cantidad, si el café lleva o no azúcar o leche, de qué tipo es el helado... Pero la intención de la pregunta no era obtener una respuesta numéricamente precisa, sino conocer hasta qué punto nuestros alumnos hacían uso de la energía de tipo químico en sus explicaciones ante un problema en donde intervenía también la energía térmica. Como datos orientativos, un helado aporta unos $8,5 \mathrm{~kJ}$ por gramo, lo cual supone unos $425 \mathrm{~kJ}$ en una ración de $50 \mathrm{~g}$. En cuanto al café, su aporte energético como alimento depende de sus posibles «añadidos», principalmente del azúcar. Así, un café con una cucharada de $10 \mathrm{~g}$ de azúcar aportaría unos $156 \mathrm{~kJ}$ (datos tomados de Mataix Verdú 1991). En cuanto a la energía asociada a «estar caliente», la diferencia de energía entre 
un vaso de café $\left(100 \mathrm{~cm}^{3}\right)$ caliente $\left(67^{\circ} \mathrm{C}\right)$ y a la temperatura de nuestro organismo $\left(37^{\circ} \mathrm{C}\right)$ supone unos $12 \mathrm{~kJ}$.

\section{RESULTADOS Y ANÁLISIS}

\section{Asociación de palabras}

En el test de asociación de palabras, los 92 alumnos escribieron un total de 627 palabras asociadas a energía, lo cual supone una media de 6,84 palabras por alumno. La palabra más frecuentemente citada fue «fuerza», con 71 menciones, seguida de "calor» con 64 y «luz» con 51 menciones. El número total de palabras diferentes recogidas en el test fue de 158 (Tabla III).

Tabla III

Palabras más citadas por alumnos de Magisterio en el test de asociación de palabras.

\begin{tabular}{lcc}
\hline Palabra & $\begin{array}{c}\text { Núm. de altumnos que } \\
\text { la citan }(\mathrm{N}=92)\end{array}$ & $\begin{array}{c}\text { \% de alumnos } \\
\text { que la citan }\end{array}$ \\
Fuerza & 71 & 77 \\
Calor & 64 & 70 \\
Luzz & 51 & 55 \\
Sol & 42 & 46 \\
Trabajo & 35 & 38 \\
Movimiento & 22 & 24 \\
Vida & 22 & 24 \\
Potencia & 20 & 22 \\
Ternperatura & 17 & 18 \\
Vitalidad & 16 & 17 \\
Total: & 158 palabras & 627 citas \\
\hline
\end{tabular}

Las palabras fueron clasificadas en dos grupos, según fueran o no clasificables por núcleos temáticos de las disciplinas científicas. En el grupo de palabras clasificables según núcleos temáticos de las ciencias se establecieron seis categorías: mecánica, luz, termodinámica, electricidad, química y nuclear. En el grupo restante se establecieron otras cinco categorías: vitalidad, vida, materia, técnica y «sin clasificar».

En la tabla IV se refleja el número de palabras incluidas en cada categoría, así como los dos términos más frecuentes en cada una de ellas, seguidos, entre paréntesis, del número de apariciones. En la categoría que denominamos "Química» aparecen reflejadas todas las palabras que hemos incluido en la misma.

De la observación de los resultados de la prueba de asociación de palabras destaca el elevado número de referencias a la Mecánica (palabras como fuerza, trabajo, movimiento...), que representan casi la tercera parte del total, siendo también muy importantes las asociaciones con la Termodinámica (calor, temperatura...) y con la luz y el Sol, éstas últimas sobre todo en los alumnos y alumnas de Magisterio de la especialidad de preescolar.
Es de destacar el escasísimo número de referencias a la energía química, apareciendo referencias al carbón $(0,3 \%)$, al petróleo $(0,3 \%)$ o a la alimentación $(1,4 \%)$, pero estando totalmente ausentes referencias a combustibles tan cotidianos como el butano o la gasolina o a sustancias como la glucosa, el azúcar o las grasas. Esto nos lleva a pensar en la escasa importancia que se le concede al aspecto energético de los combustibles y los alimentos en el currículoescolar a pesar de ser aspectos tan importantes y tan cercanos en la vida diaria.

Resulta también llamativo, el número de palabras asociadas a «vitalidad», con términos como la misma palabra vitalidad o salud, vigor..., que corresponden a un significado aceptado del término energía por el diccionario de la Real Academia, pero que suponen una concepción alternativa del concepto científico de energía.

\section{Test de lápiz y papel}

Las respuestas de los estudiantes fueron divididas en tres grupos: café, helado e igual o depende, incluyendo en este último grupo las contestaciones de alumnos que señalaban en la explicación la necesidad de conocer o especificar la composición de los sistemas «café caliente» y «helado» para poder optar por alguno de ellos.

Dentro de los tres grupos anteriores, las contestaciones a la parte abierta de la pregunta han sido clasificadas como aceptables o alternativas en función de los marcos conceptuales de los alumnos. La clasificación en categorías se realizó inicialmente de forma separada por los dos autores, obteniéndose una concordancia del $88 \%$. Los casos no concordantes fueron revisados antes de obtener el resultado final de forma consensuada.

Hemos incluido en la categoría de aceptable aquellas respuestas en que los alumnos tienen en cuenta en sus razonamientos Ios contenidos en energía química de las distintas sustancias que creen que están presentes en el helado o en el café, bien citándolas (contiene azúcar, grasas...), o bien refiriéndose a su mayor contenido energético (tiene más calorías, aporta kcal...). Creemos que esta consideración puede parecer demasiado amplia, pero la razón de hacerio así es fundamentalmente por la diversidad de niveles de enseñanza que recoge la prueba, pues si bien en los alumnos universitarios el nivel de exigencia podría ser mayor, consideramos que para los alumnos de EGB y BUP era conveniente dicha amplitud.

Los resultados obtenidos en los diferentes niveles se resumen en la tabla $\mathrm{V}$.

\section{Descripción de las diferentes categorías}

Entre las explicaciones que hemos considerado «aceptables» podemos destacar a modo de ejemplos. (Las respuestas de los alumnos aparecen reflejadas textualmente):

«Yo creo que el que aporta más energía es el helado, debido a las sustancias de qué está formado, es decir, 
Tabla IV

Contestaciones de estudiantes de $3 r$ curso de Magisterio (especiatidades de Ciencias y Preescolar) al test de asociación libre de palabras. $3^{2}$ de Preescolar: 52 alumnos, 362 palabras. $3^{2}$ de Ciencias: 40 alumnos, 265 palabras. Total: 92 alumnos, 627 palabras ( 6,84 palabras por alumno).

Categorias

Mécanica

Luz

Termodinámica

Vitalidad

Electricidad

Vida

Materia

Técnica

Química

Nuclear

Otros
$3^{9}$ de Preescolar

Fuerza (45),

Potencia (11),

Luz (40),

Sol (38),

Calor (26),

Caloria(s) (2),

Vitalidad (9),

Salud (4).

Electricidad (7),

Eléctrica (3),

Vida (20),

Animal (1),

Agua (12),

Materia (6),

Motor (3),

Automoción (1),

Comida (4), Carbón (2),

Cola Cao (2), Petró.

leo (2), Alimentación (I),

Carbono (I), Química (1).

Nutricion (1), Nutritiva (1).

Nuclear (3),

Atómica (1).

Ciencia (4),

Física (2),
$3^{2} \mathrm{de}$ Ciencias

Total

87

Trabajo (32),

Fuerza (26),

85

Luz (11),

Sol (4),

33

Calor $(38)$,

Temperatura (17).

32

Vitalidad (7),

Vigor (1),

15

Corriente (4),

Electricidad (6),

22

Vida (2),

Nacimiento (2),

21 Agua (1),

Aire (1),

13 Industria (7),

Tecriología (1),

is

Fuego (2), Combus-

tión (l), Llama (l),

Reacciones

químicas (1).

\section{4}

Atómica (1),

35 Universo (2),

Cambios (1).
105

19

63

16

2

6

192

104

96

48

28

28

26

24

20

20

55

\section{Tabla V}

Resultados de Jas contestaciones al test de lápiz y papel.

Contestación

EGB (101) BUP (22) EU (44) CAP (16)

Contestación

EGB (101) BUP (22) EU (44)

CAP (16)

\section{Aporta más energía}

el helado.

Aceptable

Alternativa

Sin explicación o

incoherente

Aporta más energía

el café.

Aceptable

Altemativa

Sin explicacion o

incoherente

$\begin{array}{rrrr}57 & 10 & 27 & 14 \\ 33 & 6 & 26 & 14 \\ 19 & 2 & 1 & 0 \\ 5 & 2 & 0 & 0\end{array}$

39

10

24

5
Aportan igual energía

o depende.
Aceptable
Alternativa
Sin explicación
o incoherente

$\begin{array}{rrrr}\mathbf{5} & \mathbf{5} & \mathbf{1 2} & \mathbf{2} \\ 1 & 2 & 7 & 2 \\ 2 & 3 & 1 & 0 \\ 2 & 0 & 4 & 0\end{array}$


debido a su composición, azúcar, glucosa, colorantes, etc. Esas sustancias tienen muchas Kcal.» (2 BUP).

«Un vaso de café muy caliente porque tiene azúcar y el helado tiene agua» (EGB).

"Creo que un vaso de café muy caliente aporta calorías pero un helado también las aporta y según el helado que sea puede aportar más o menos» (EGB).

«El helado aporta al organismo más calorías, por lo tanto más energía» (CAP).

Es un hecho destacable que la mayoría de las explicaciones que dan los alumnos del CAP, licenciados en Química, se parecen mucho a las que podría dar una persona no especialista en el tema, como la del ejemplo anterior. Solamente una contestación incluye otras ideas provenientes de la instrucción formal, aunque globalmente no se pueda calificar de correcta sin ulteriores precisiones:

«La energía aportada por un alimento a un organismo se debe a la diferencia energética entre las moléculas de ATP que produce y la energía que hay que suministrar para que el organismo lo asimile. El helado produce más ATP debido a que tiene más grasas e hidratos de carbono que el café. No tiene nada que ver la temperatura a la que se encuentre» (CAP).

Esta dificultad para trasladar los conocimientos aprendidos en clase a situaciones de la vida cotidiana aparece reflejada en los trabajos de Black y Solomon (1983) y Solomon (1983).

Dentro de las contestaciones clasificadas como alternativas son frecuentes las que razonan únicamente en términos de calor aportado. Son elevados los porcentajes de alumnos que piensan que el café proporciona más energía al organismo que el helado por estar caliente, es decir, "ven» la energía térmica como "verían" con claridad la energía potencial gravitatoria o la cinética, pero no «ven» la energía química del helado. Nuestra experiencia con alumnos de Magisterio es que quedan verdaderamente sorprendidos cuando realizan experiencias en que se mide la energía que desprende $1 \mathrm{~g}$ de alcohol (etanol) al quemarse (algunos incluso proponen la utilización del alcohol como combustible en cocinas, calefacciones, etc.), ya que les cuesta imaginarse que una cantidad tan pequeña de combustible llegue a desprender tal cantidad de energía.

Algunos ejemplos de estas explicaciones son:

«Un vaso de café caliente. Porque el calor aporta más energía al cuerpo que el frío» (EGB).

«Un vaso de café muy caliente. Porque hay una transmisión de E del vaso de café hacia el organismo. En el caso del helado es el organismo el que aporta energía» (EU).

Otras interpretaciones alternativas frecuentes son aquéllas que aluden a sensaciones de bienestar o incluso a épocas del año:
«Depende, si hace frío, un vaso de café caliente, porque tomándolo subirá la temperatura del cuerpo y ya no tendrás tanto frío. Si hace calor, un helado porque te refrescará y no sentirás la pesada calor, así tenơrás mas energía y podrás hacer más cosas» ( $2^{\circ}$ de BUP).

«Cualquiera de las dos cosas, lo que pasa es que el helado (sic) es para el verano, y el café más bien para el invierno, según lo tomes en esas fechas te aportará más energía ( $2^{2}$ de BUP).

Otro grupo de contestaciones alternativas lo constituyen aquéllas que atribuyen el mayor o menor aporte energético de un alimento a su contenido en vitaminas (mayoritariamente en el helado), o aquellas otras que lo asocian al efecto estimulante de la cafeína:

«Un helado, porque tiene muchas vitaminas y no te quita el sueño como el café» (EGB).

«Un vaso de café muy caliente. Porque el café tiene cafeína y te despierta, te da energía» (EGB).

Algunos alumnos consideran sin embargo este efecto estimulante en sentido contrario:

«Un helado, porque el café contiene mucha cafeína y puede producirte nerviosismo, o te quita el sueño» (EGB).

\section{IMPLICACIONES PARA LA ENSEÑANZA- APRENDIZAJE DE LAS CIENCIAS}

La estructuración clásica de los conceptos científicos según la lógica interna de las distintas disciplinas que se refleja en la mayoría de los diseños curriculares actuales, como indica Pozo (1987), no siempre se corresponde con la estructuración psicológica de dichos conceptos en la mente de los alumnos. Lo que para los profesores de Física y Química y de Ciencias Naturales es «evidente» parece que puede no serlo tanto para los alumnos, como muestran los resultados obtenidos en este trabajo y como ya apuntaba Gayford (1986) acerca del concepto de Energía. Se puede «enseñar» la energía química en la asignatura de Física y Química y enseñar temas de Biología, como la producción de materia orgánica por los productores primarios o temas de nutrición..., que se apoyan en conceptos energéticos, y el resultado puede ser que los alumnos no sean capaces de conectar unos conocimientos con ios otros, volviendo a utilizar conocimientos de tipo «dietético» obtenidos, la mayor parte de las veces, de fuentes «no formales» como los medios de comunicación... Otra alternativa es buscar una aproximación más coordinada a este aspecto de la enseñanza, como propone Wood-Robinson (1986): «No hay duda de que es necesaria una aproximación más coordinada al concepto de energía entre los profesores de ciencias desde la física a la biología».

Una estructuración de tipo disciplinar como la actual puede presentar los mismos conceptos de forma que a los 
alumnos les parezcan diferentes y no se produzca la conexión mental. Podría ser que desde una estructuración de Area de Ciencias (Jiménez Aleixandre, GarcíaRodeja y Lorenzo Barral 1991), como la que propone el DCB, la cooperación entre los profesores de Física y Química y Ciencias Naturales se vea favorecida al tener que compariir el mismo diseño curricular, y sea fructífera sobre la enseñanza-aprendizaje de aspectos como el de la energía.

Por otro lado nos volvemos a ratificar en lo dicho en otros trabajos (Lorenzo Barral 1990) sobre la importancia que tiene para los profesores el «ser conscientes de las dificultades de los alumnos en el aprendizaje de contenidos concretos». En este sentido tenemos que destacar que ya hay muchos materiales curriculares, incluyendo libros de texto, que tienen en cuenta las dificultades de
Ios alumnos, aunque esta consideración casi siempre se restringe a los temas de mecánica. La energía química es difícil de entender y por tanto sería conveniente dedicarle mayor esfuerzo por parte de profesores y alumnos.

\section{AGRADECIMIENTOS}

Los autores agradecen a Marfa Pilar Jiménez Aleixandre sus sugerencias en la preparación de la versión escrita de este trabajo. J.R.G.O. agradece a la Consellería de Educación (Xunta de Galicia) la concesión de una ulicencia por estudios» que le ha permitido la realización de este trabajo. F.M.L.B. agradece a la Xunta de Galicia la financiación del proyecto XUGA 7180189, del cual forma parte la investigación presentada en este trabajo.

\section{REFERENCIAS BIBLIOGRÁFICAS}

BLACK, P. y SOLOMON, J, 1983. Life world and science world pupils ideas about energy, en Marx, G. (ed.), Entropy in the school, Vol. 1. (Roland Eotvos Physical Society: Budapest, Hungría).

BROOK, A., 1986. Childrens' understanding of ideas about energy: a review of the literature, en Driver, $R$. y Millar, $R$. (eds.), Energy Matters. (Leeds: Gran Bretaña)

DAPENA MÁRQUEZ, J.M., BECERRA GIL, F. y COROAS BARGUEIRAS, M., 1991. A Enerxía, en Exemplificacións didácticas educación secundaria obrigatoria. Tomo IV. (Consellería de Educación e Ordenación Universitaria. Xunta de Galicia).

GAYFORD, C.G., 1986. Some aspects of the problems of teaching about energy in school biology, European Journal of Science Education, Vol. 8, pp. 443-450.

JIMÉNEZ ALEIXANDRE, M.P., GARCIA-RODEJA, I. y LORENZO BARRAL, F.M., 1991. Pero jexiste el área de Ciencias?, Cuadernos de Pedagogia, 188, pp. 64-66.

LORENZO BARRAL, F.M., 1990. ¿Cómo flotan los cuerpos que flotan? Concepciones de los estudiantes, Enseñanza de las Ciencias, Vol. 8(3), pp. 244-250.
MATAIX VERDÚ, J., 1991. Alimentación familiar. Guía cientifico-práctica. (Escuela de nutrición: Granada).

PINTÓ CASULLERAS, M.R., 1991. Algunos conceptos implícitos en la $I^{a}$ y la $2^{a}$ Leyes de la Termodinámica: una aportación al estudio de las dificultades de su aprendizaje. Tesis doctoral. (Universidad Autonoma de Barcelona).

POZO, J.I., 1987. Aprendizaje de la Ciencia y Pensamiento Causal. (Visor: Madrid).

SHAVELSON, R.J., 1974, Methodis for examining representations of a subject-matter structure in a student's memory, Journal of Research in Science Teaching, Vol. 11, pp. 231-249.

SOLOMON, J., 1983. Leaming about energy. How pupils think in two domains, European Journal of Science Education, Vol. $5, \mathrm{pp}$. 49-59.

WELCH, W.W., 1986. Leaming about energy: a review of the literature, Working poper, 301. (SERU, University of Waikato: Hamilton, Nueva Zelanda).

WOOD-ROBINSON, C., 1986. Energy - a biologist's viewpoint, en Driver, R. y Millar, R. (eds.), Energy Matters. (Leeds: Gra Bretaña). 